\title{
Influenza A (não H1N1) associada à insuficiência respiratória e renal aguda em paciente com fibrose cística previamente vacinado: relato de caso.
}

Louise Piva Penteado ${ }^{\text {; }}$ Cecília Susin Osório² ; Paulo de Tarso Roth

Dalcin ${ }^{3}$.

\section{Resumo}

INTRODUÇÃO: A infecção por influenza A é uma doença prevalente em todo o mundo, sendo o subtipo H3N2 o mais encontrado. Espera-se, no entanto, que indivíduos vacinados não sejam infectados. Complicações, como insuficiência respiratória aguda e insuficiência renal aguda podem ser atreladas a ela. Esse relato busca elucidar um caso de influenza A (não H1N1) com complicações em paciente com doença pulmonar crônica vacinado.

MÉTODOS: Os dados sobre o paciente foram obtidos em prontuário eletrônico do Hospital de Clínicas de Porto Alegre, com o consentimento deste e do paciente.

RELATO DE CASO: Paciente masculino, 39 anos, com fibrose cística, vacinado contra a gripe com vacina tríplice apresentou caso de infecção por influenza A (não H1N1), em junho de 2015 na cidade de Porto Alegre - RS. Evoluiu para insuficiência respiratória aguda, tendo que ser submetido à ventilação mecânica e cuidados intensivos. Apresentou também insuficiência renal aguda, sendo submetido à diálise. Recebeu alta, após ciclos de antibióticos, ventilando espontaneamente e função renal normal.

RESULTADOS/DISCUSSÃO: A infecção por influenza A, associada à doença de base, precipitou a insuficiência respiratória aguda. $\mathrm{O}$

\footnotetext{
${ }^{1}$ Acadêmica de medicina da Universidade Federal do Rio Grande do Sul (UFRGS), bolsista de iniciação científica PIBIC UFRGS. (Ipenteado@hcpa.edu.br)

${ }^{1}$ Acadêmica de medicina da Universidade Federal do Rio Grande do Sul (UFRGS), bolsista de iniciação científica BIC UFRGS. (cecilia.susin@gmail.com)

${ }^{3}$ Pneumologista, Serviço de Pneumologia do HCPA; professor associado, Departamento de Medicina Interna, Faculdade de Medicina, UFRGS.
} 
paciente provavelmente foi infectado com o vírus influenza A subtipo H3N2, pois, segundo boletim epidemiológico, o vírus circulante mais prevalente no período foi o $\mathrm{H} 3 \mathrm{~N} 2$ e a secreção de nasofaringe deu positiva para influenza A sazonal e negativa para H1N1. Contudo, de acordo com a Anvisa essa cepa estava incluída da vacina de 2015. O CDC publicou em dezembro de 2014 informações sobre discordância antigênica entre cepa circulante e cepa da vacina. A insuficiência renal aguda tem pouca evidência de associação com influenza, contudo têm-se alguns relatos e prováveis causas.

CONCLUSÃO: A vacina tríplice antigripal do ano de 2015 pode não ter coberto a cepa de influenza A H3N2 que infectou o paciente. Além disso, o paciente apresentou insuficiência renal aguda, não comumente associada à infecção por influenza.

Palavras Chave: influenza A; insuficiência respiratória aguda; insuficiência renal aguda; fibrose cística.

\section{Introdução}

A influenza ou gripe é uma doença respiratória aguda e contagiosa, causada pelo vírus da influenza A ou B. Estima-se que $10 \%$ da população mundial apresenta ao menos um episódio anual de influenza (WHO, 2016). Pacientes com doença crônica, em especial doença pulmonar crônica, são mais suscetíveis às complicações graves causadas pela gripe.

Em dezembro de 2014, o Center of Disease Control (Estados Unidos) relatou que a atividade pela influenza no hemisfério norte era causada principalmente pelo vírus da influenza A H3N2 (Journal Watch NEJM, 2014; CDC, 2016). No entanto, a vacina não abrange todas as cepas (Adler, 2015).

A insuficiência renal aguda não é uma complicação frequente e, quando isto ocorre, está relacionado à rabdomiólise. (Foulkes, 1990, p.303-4; Leebeek, 1995, p.189192; Dell, 1997, 363-5; Nauss, 2009, 372.e5)

O presente trabalho tem por objetivo relatar um caso de influenza A sazonal (não H1N1), ocorrido em junho de 2015, em paciente com fibrose cística, submetido à vacinação antigripal trivalente em abril de 2015, que evoluiu com insuficiência respiratória aguda e insuficiência renal aguda sem rabdomiólise. 


\section{Métodos}

Esse relato de caso foi elaborado a partir de dados coletados e registrados em prontuário eletrônico pela equipe da pneumologia e da nefrologia do Hospital de Clínicas de Porto Alegre. Contatou-se com os médicos responsáveis pelo caso para acessar as informações. O paciente deu consentimento ao registro e publicação.

\section{Relato de caso}

\subsection{Identificação}

Paciente masculino de 39 anos, procedente do Rio Grande do Sul.

\subsection{Queixa principal}

Piora do quadro respiratório.

\subsection{História da doença atual}

Diagnosticado com fibrose cística aos nove anos de idade, com mutação em delta F508 (2 alelos - homozigoto). Atualmente apresenta distúrbio ventilatório moderado, bronquiectasias, colonização crônica por Pseudomonas aeruginosa e insuficiência pancreática exócrina. Chegou ao hospital com piora do quadro respiratório e prostração nos dias prévios à internação. Relata que familiares apresentavam sintomas respiratórios condizentes com quadro gripal.

\subsection{Evolução}

Ao exame físico da chegada, encontrava-se em regular estado geral, dispneico, acianótico, com crepitantes difusos, sem sibilos, aumento do tempo expiratório, saturando $50 \%$ em ar ambiente e temperatura axilar de $37,1^{\circ} \mathrm{C}$. À primeira impressão, fez-se diagnóstico de broncopneumonia como causa da exacerbação da fibrose cística, tratada com piperacilina/tazobactam e tobramicina, conforme colonização prévia de janeiro de 2015 - Pseudomonas aeruginosa.

Evoluiu para insuficiência respiratória aguda concomitante com desenvolvimento de insuficiência renal aguda (creatinina de 2,37 sendo que o basal era 0,79) e hipercalemia (secundária a perda da função renal e acidose respiratória). A secreção da nasofaringe foi negativa para o vírus influenza A H1N1 e positiva para vírus da influenza A sazonal. Nesse momento, foi associado oseltamivir ao tratamento, menos de 24 horas da suspeita de gripe. O paciente seguiu hipoxêmico, com saturação de $56 \%$ em ventilação não invasiva. Foi então encaminhado para UTI e submetido a intubação orotraqueal.

Além disso, evoluiu com agravamento da função renal, apresentando creatinina de 5,87 e estado anúrico. Iniciou hemodiálise contínua. Em virtude da persistência do quadro e considerando choque séptico com foco pulmonar, foram associados os 
antibióticos ciprofloxacino e vancomicina. Sete dias após a entrada no hospital pode ser extubado e iniciou diálise intermitente, recebendo alta da UTI.

Obteve melhora parcial do quadro pulmonar na enfermaria e após uma semana retornou a UTI com suspeita de tromboembolismo pulmonar (TEP) ou nova infecção respiratória. Foi realizada angio-tomografia que excluiu a possibilidade de TEP. Iniciou novo curso de antibióticos e teve excelente resposta clínica. A insuficiência renal aguda foi resolvida. Atualmente, o paciente encontra-se em reabilitação pulmonar.

\subsection{Exames complementares}

A primeira radiografia da internação apresentava fibroatelectasias antigas, relacionáveis a fibrose cística, áreas de consolidação alveolar e extensas na metade inferior do pulmão esquerdo e bronquiectasias contendo grande quantidade de líquido em seu interior no lobo superior à direita. Obstrução do seio costofrênico lateral à direita por espessamento e/ou derrame pleural (figura 1).

A angio-tomografia, realizada quando houve suspeita de tromboembolismo pulmonar, não identificou defeito de enchimento na circulação arterial pulmonar sugestivo de tromboembolismo pulmonar agudo ou crônico; extensas manifestações de doença das vias aéreas, compatíveis com fibrose cística; atualmente com sinais de infecção sobreposta, particularmente em lobo inferior esquerdo; presença de escavações confluentes no ápice pulmonar direito (Figura 2).

Figura 1: Radiografia do paciente no dia seguinte à baixa.

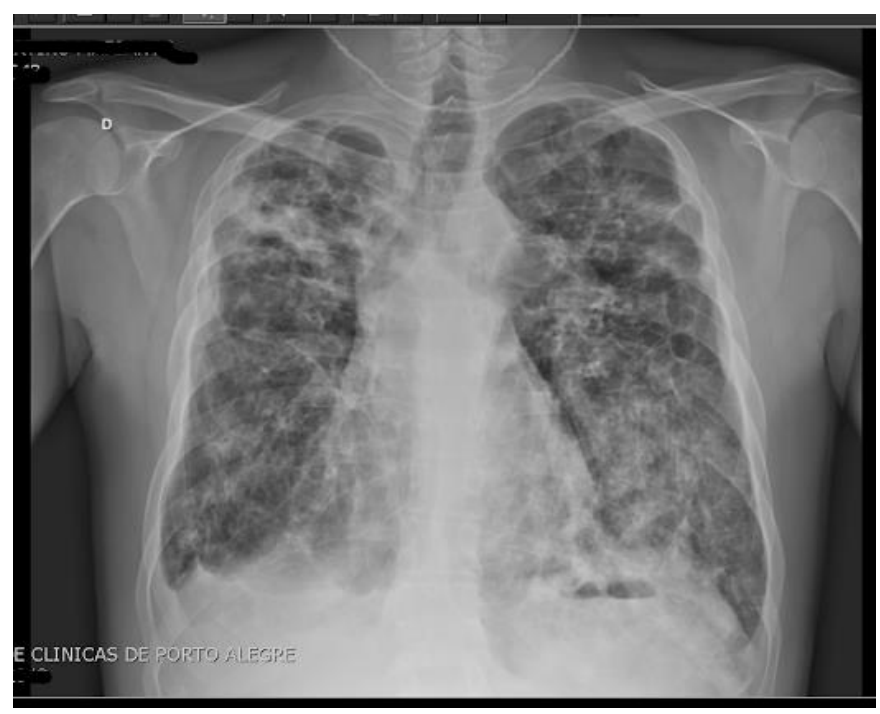

Fonte: prontuário eletrônico do HCPA

Figura 2: Cortes de Angio-tomografia realizada 21 dias após a internação. A: segmento médio (alterações da fibrose cística); B: lobo inferior esquerdo (processo infeccioso). 


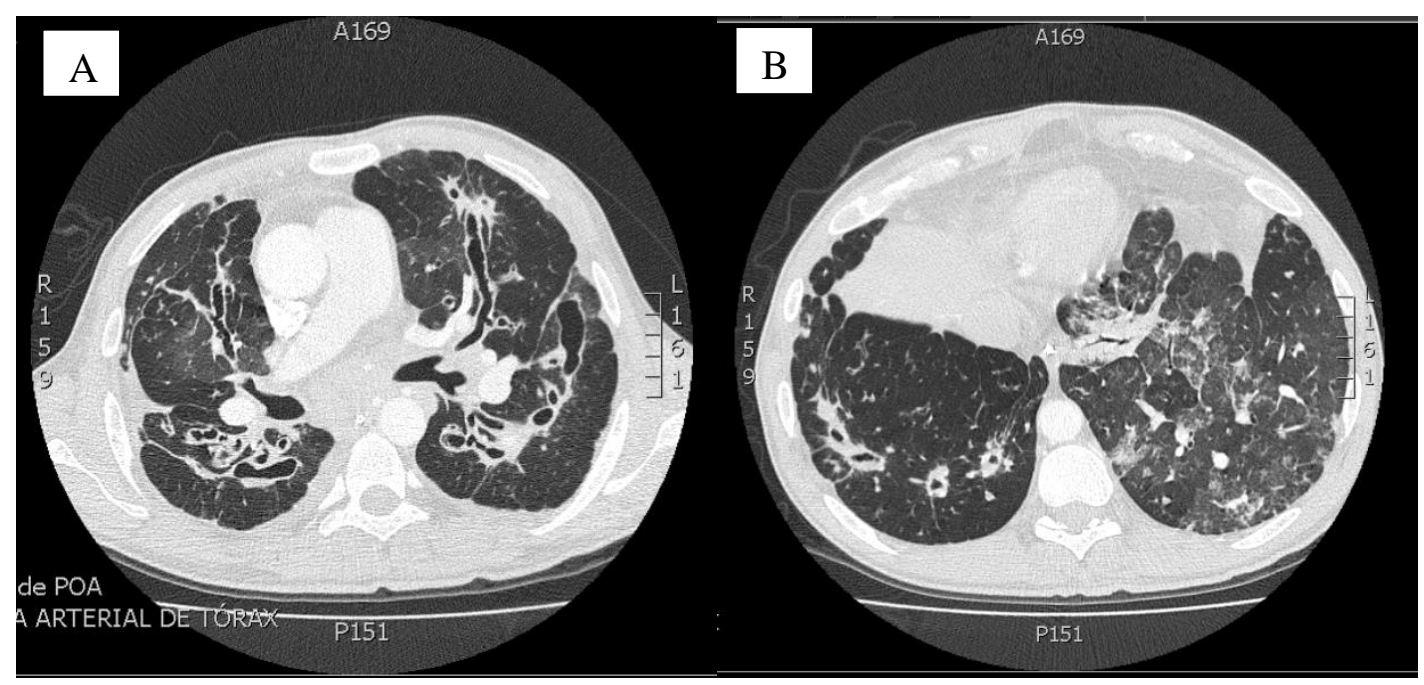

Fonte: prontuário eletrônico do HCPA

\section{Resultados/Discussão}

O paciente, após a infecção por influenza, desenvolveu pneumonia bacteriana. $\mathrm{O}$ que poderia ser esperado, uma vez que "a complicação mais frequente de influenza é pneumonia, mais comumente pneumonia bacteriana secundária (ex: Streptococcus pneumoniae, Haemophilus influenzae ou Staphylococcus aureus)" (The Pink Book: Course Textbook, 2015, p.190).

Entretanto, o paciente não era previamente hígido, por ter fibrose cística, o que precipitou a insuficiência respiratória aguda apresentada após a infecção. Tal afirmação se torna o pilar desse relato, uma vez que o paciente recebe a vacina tríplice contra a gripe anualmente e mesmo assim foi infectado pelo vírus, tendo que ser submetido a cuidados de tratamento intensivo e ventilação mecânica para reverter o agravamento do quadro pulmonar.

A Coordenação Geral de Doenças Transmissíveis do Ministério da Saúde monitora os dados epidemiológicos da influenza no país, com a elaboração de Boletins Epidemiológicos que são encaminhados às equipes técnicas da vigilância dos estados e colaboradores, para ampla divulgação.

Segundo o boletim do ano de 2015, no Brasil,

Até a SE (semana) 52 de 2015 foram notificados 14.432 casos de SRAG*, sendo 11.945 (82,8\%) com classificação final. Destas, 9,1\% (1.089/11.945) foram classificadas como SRAG por influenza e 22,5\% (2.685/11.945) como outros vírus respiratórios. Dentre os casos de influenza 599 (55,0\%) eram influenza A(H3N2), 234 (21,5\%) influenza B, 141 (12,9\%) 
A(H1N1) e 115 (10,5\%) influenza A não subtipado. (Portal da Saúde, boletim semana 52, 2016, p.1)

*SRAG (Síndrome Respiratória Aguda Grave): indivíduo hospitalizado com febre, acompanhada de tosse ou dor de garganta e que apresente dispneia. Também podem ser observados os seguintes sinais: saturação de $\mathrm{O} 2$ menor que $95 \%$ ou desconforto respiratório ou aumento da frequência respiratória.

O teste para investigação de influenza humana em secreção de nasofaringe indicou que a amostra era negativa para influenza A (H1N1) e positiva para vírus influenza A sazonal. Dessa forma, acredita-se que o vírus infectante seria o H3N2. Contudo, a Anvisa (Agência Nacional de Vigilância Sanitária) publicou no Diário Oficial da União de outubro de 2014 que a vacina contra influenza no ano de 2015 conteria as seguintes cepas de influenza: "um vírus similar ao influenza A H1N1, um vírus similar ao vírus influenza A H3N2 e um vírus similar ao influenza B" (DOU outubro de 2014, 2014, p.68).

O CDC noticiou em dezembro de 2014 e janeiro de 2015 que metade dos vírus testados não respondeu à vacina antigripal (WHO, 2016), pois 67\% dos vírus identificados eram diferentes antigenicamente daqueles incluídos na vacina. (Adler, 2015)

O paciente apresentava também insuficiência renal aguda associada à infecção por influenza A. Quatro causas potenciais desse quadro foram encontradas: rabdomiólise, injúria viral direta ao vírus, hipoperfusão renal levando à sepse e coagulação intravascular disseminada (Watanabe, 2003, p.543).

Ainda segundo o autor,

Uma vez que envolvimento renal em pacientes com infecção pelo vírus influenza A é incomum, na maioria das vezes sendo reportada como caso único ou uma série pequena de pacientes, as características clínicas da injúria renal em pacientes com infecção por influenza A permanecem obscuras. (Watanabe, 2003, p.543)

\section{Conclusão}

Esse relato elucida que a vacina tríplice antigripal do ano de 2015 pode não ter coberto a cepa de influenza A H3N2 que infectou o paciente, considerando provável diferença antigênica entre a cepa presente na vacina e a cepa circulante no referido período. Ademais, o paciente apresentou insuficiência renal aguda, não comumente associada à infecção por influenza, com poucas descrições prévias. 


\title{
Influenza $A$ (not H1N1) associated with acute respiratory and renal failure in patient with cystic fibrosis previously vaccinated: a case report
}

\begin{abstract}
Introduction: The influenza A infection is a prevalent disease in the whole world, and the subtype H3N2 is the most commonly found. However, it is expected that individuals vaccinated will not be infected. Complications, as acute respiratory failure and acute renal failure, can be linked to it. This report aims to elucidate a case of influenza A (not H1N1) with complication in a patient with chronic lung disease previously vaccinated.
\end{abstract}

Methods: The patient data were collected in electronic medical records of Hospital de Clínicas de Porto Alegre, with hospital and patient's consent.

Case report: Male patient, 39 years old, with cystic fibrosis, vaccinated with triplex vaccine against influenza, presented an influenza (not H1N1) infection, in June 2015 in Porto Alegre - RS. He evolved to acute respiratory failure, being submitted to mechanical ventilation and intensive care. He also presented acute renal failure, being 
submitted to dialysis. He was discharged after antibiotics cycles, spontaneously breathing and with normal renal function.

Results and Discussion: The influenza A infection, associated with cystic fibrosis, induced the acute respiratory failure. The patient probably was infected with influenza A virus subtype H3N2, because according to epidemiological bulletin the prevalent virus in this period was H3N2 and the nasopharyngeal secretion was negative for H1N1. According to Anvisa, this strain was included in the 2015 vaccine. The CDC published in December 2014 information about the antigenic difference between the current strain and the vaccine strain. There is few information about the association of the acute renal failure and the influenza infection, however there are some reports and probable causes.

Conclusion: The 2015 triplex vaccine against influenza may not have covered the strain of influenza A H3N2 that infected the patient. Besides that, the patient presented acute renal failure, not commonly associated with influenza infection.

Keywords: Influenza A. Acute respiratory failure. Acute renal failure. Cystic fibrosis.

\section{Referências}

ADLER, Cara. This Season's Flu Vaccine Just 23\% Effective. Jornal Watch - New England Jornal of Medicina, Massachussets, 16 jan. 2015. Disponível em: < http://www.jwatch.org/fw109751/2015/01/16/seasons-flu-vaccine-just-23effective> Acesso em: 1 fev. 2016.

BRASIL. MINISTÉRIO DA SAÚDE. SECRETARIA DE VIGILÂNCIA EM SAÚDE. BOLETIM EPIDEMIOLÓGICO. Influenza: Monitoramento até a Semana Epidemiológica 52 de $2015 . \quad$ Disponível em: <http://portalsaude.saude.gov.br/images/pdf/2016/fevereiro/25/Boletim-Epidemiol-gico-Influenza-SE52-2015.pdf> Acesso em: 1 fev. 2016.

BRASIL. Resolução RDC n. 62, de 16 de outubro de 2014. Dispõe sobre a composição das vacinas influenza a serem utilizadas no Brasil no ano de 2015. Diário Oficial da União, Brasília, n. 201, p. 68, 17 out. 2014. Seção 1.

CENTER OF DISEASE CONTROL AND PREVENTION - CDC. The Pink Book: Course Textbook - 13th Edition (2015). Vaccines and Immunizations - Influenza - aEpidemiology and Prevention of Vaccine-Preventable Diseases. Disponível 
em: < http://www.cdc.gov/vaccines/pubs/pinkbook/flu.html\#complications> Acesso em: 2 fev. 2016.

\section{CENTER OF DISEASE CONTROL AND PREVENTION - CDC. What You Should}

Know for the 2014-2015 Influenza Season. Disponível em: <http://www.cdc.gov/flu/pastseasons/1415season.htm> Acesso em: 2 fev. 2016.

DELL, Katherine Mac Era; SCHULMAN, Seth L. Rhabdomyolysis and acute renal failure in a child with influenza A infection. Pediatric Nephrology, v. 11, p. 363-5, 1997.

FOULKES, William; REES, Jeremy; SEWRYT, Caroline. Influenza A and rhabdomyolysis. Journal of infection., v. 21, p. 303-4, nov. 1990.

LEEBEEK, F.W.G.; et al. Rhabdomyolysis associated with influenza A vírus infection. Netherlands Journal of Medicine, v. 46, p. 189-192, 1995.

NAUSS, Michael D.; SCHMIDT, Erin L.; PANCIOLI, Arthur M. Viral myositis leading to rhabdomyolysis: a case report and literature review. American Journal of Emergency Medicine, v. 27, p. 372.e5-372.e6, mar. 2009.

U.S. Flu Activity on the Rise, but Still Low. Jornal Watch - New England Jornal of Medicina, Massachussets, 19 dez. 2014. Disponível em: <http://www.jwatch.org/fw109658/2014/12/19/us-flu-activity-rise-still-low> Acesso em: 1 fev.2016.

WATANABE, TORU; et al. Renal involvement in children with influenza A vírus infection. Pediatric Nephrology, v. 18, p. 541-4, abr. 2003.

WORLD HEALTH ORGANIZATION - WHO. Influenza. Disponível em: <http://www.who.int/biologicals/vaccines/influenza/en/> Acesso em: 2 fev. 2016. 
